\title{
Long-term follow-up of photocoagulated retinal breaks
}

\author{
M. ROMEM AND L. SINGER \\ From the Department of Ophthalmology, Jaffo Government Hospital and School of Medicine, \\ Tel Aviv University, Israel
}

SUMMARY 126 cases of retinal breaks treated in our department by photocoagulation during a period of 10 years (1963-73) were selected for a close annual follow-up. Eliminated from the study were cases of macular holes, breaks which developed in an eye after retinal detachment surgery, and cases in which a close follow-up was impossible for various reasons.

The study includes symptomatic retinal breaks in myopic, non-myopic, and aphakic eyes as well as asymptomatic holes which were found in the undetached fellow eye. Four eyes treated by photocoagulation $(3.2 \%)$ developed a retinal detachment. The causes of failures and complications, such as retinal detachment, vitreous haemorrhage, and macular pucker, are discussed.

The prophylactic treatment of retinal breaks has been a subject of longstanding controversy (Chignell and Shilling, 1973). While some years ago prophylactic treatment was considered justified only in exceptional cases (Byer, 1967), recently all retinal breaks are being treated, apart from holes near the ora serrata (Kanski and Daniel, 1975; Chignell and Shilling, 1973). It has been shown that while the number of retinal detachments decreased significantly (from 10.9 to $4.2 \%$ ) in the treated retinal breaks (Merin et al., 1971), it increased in the untreated cases (Robertson, 1973).

Concerning the selection of cases it is generally agreed that symptomatic horseshoe holes and holes in an aphakic eye are more liable to develop into a retinal detachment (Lincoff, 1961; Robertson and Norton, 1973). It has also been shown that a retinal hole usually leads to a detachment only during the initial period of its existence (Neumann et al., 1972).

Photocoagulation, cryopexy, or surgical methods are at present employed (Kanski and Daniel, 1975; Chignell and Shilling, 1973), without significant differences between the result of the various procedures. It thus seems that a rational selection of cases and carefully performed procedure is the key to favourable results.

\section{Materials and methods}

We undertook to analyse a group of 126 eyes in

Address for reprints: Dr M. Romem, Department of Ophthalmology, Jaffo Government Hospital and School of Medicine, Tel Aviv University, Israel
120 patients with retinal breaks treated by photocoagulation in our department during a period of 10 years (1963 to 1973 ).

Actually, 288 eyes had been treated. For the purpose of this study only 126 eyes are included, i.e., those cases in which the follow-up lasted for at least 2 years and could be thoroughly carried out. Excluded from the study were cases in which the follow-up lasted less than 2 years, in which the data were incomplete, or the patient did not appear regularly for examinations; also excluded were cases of macular holes, breaks developing after a retinal detachment, and those cases in which a close follow-up was impossible owing to the development of opacities of the media, another illness, or death. The treated eyes were examined thoroughly at least once a year by slit-lamp biomicroscopy, using the Goldmann three-mirror contact lens. The visual acuity was documented and the location and the form of the lesion were drawn. Symptomatic and asymptomatic breaks were treated.

The symptomatic breaks included horseshoe and round holes (over 1 papillary diameter), and latticelike or other degenerative changes with 1 or more holes in myopic, non-myopic, and aphakic eyes. Asymptomatic holes in a patient presenting with retinal detachment, when discovered by routine examination in the undetached fellow-eye, were treated.

The photocoagulation was performed through the Goldmann three-mirror lens and the Fankhauser slit-lamp attached to the Zeiss-Oberkochen xenon arc photocoagulator. Topical anaesthesia only, by oxybuprocaine drops, was used in all the cases. 
When the fundus was blurred owing to a vitreous haemorrhage, treatment was withheld until the haemorrhage was absorbed.

The age, sex distribution, and the refractive errors of the patients are shown in Tables 1 and 2. The form of different breaks and their location are shown in Tables 3 and 4.

\section{Results}

In all but 4 cases the retina remained attached and a good scar developed. The visual acuity remained unchanged in all except 1 case. Retinal detachment developed in 4 cases $(3 \cdot 17 \%)$. Three of the 4 cases

Table 1 Age and sex distribution

\begin{tabular}{ll}
\hline Age & No. of cases \\
\hline $10-$ & 11 \\
$30-$ & 38 \\
$50-$ & 39 \\
$60-75$ & 38 \\
Total & 126 \\
\hline
\end{tabular}

Males $71(56 \%)$, females $55(44 \%)$

Table 2 Refractive error

\begin{tabular}{lcc}
\hline & No. & $\%$ \\
\hline Myopia & 66 & 52.4 \\
Hypermetropia & 22 & 17.5 \\
Emmetropia & 30 & 23.8 \\
Aphakia & 8 & 6.3 \\
\hline
\end{tabular}

Table 3 The form of retinal breaks

\begin{tabular}{lll}
\hline & No. & $\%$ \\
\hline Horseshoe & 73 & 57.9 \\
Round & 37 & 29.4 \\
Lattice and holes & 16 & 12.7 \\
\hline
\end{tabular}

Table 4 Location of different breaks

\begin{tabular}{lcc}
\hline & No. & $\%$ \\
\hline Upper temporal quadrant & 85 & 67.5 \\
Lower temporal quadrant & 21 & 16.7 \\
Upper nasal quadrant & 15 & 11.9 \\
Lower nasal quadrant & 5 & 4.0 \\
\hline
\end{tabular}

were fellow-eyes in patients who underwent retinal surgery in the other eye. Only 2 of these were highly myopic ( -10.0 and -20.0 dioptres). In 3 cases the retina re-attached after surgery, and 1 case developed a massive vitreous retraction after surgery.

Vitreous haemorrhage followed treatment in 5 cases; in 3 of them it preceded the development of retinal detachment and in 2 the haemorrhage was absorbed and visual acuity became normal. Macular pucker developed in 1 case, and the visual acuity decreased from $6 / 6$ to $6 / 20$.

\section{Discussion}

As mentioned above, 4 of our cases developed a retinal detachment. In 2 cases retinal detachment developed from the place of the treated breaks shortly after treatment. One of them had a horseshoe hole with a flat detached retina round the hole. This was a fellow-eye, and the retina was successfully re-attached by surgery.

The second patient had 2 large horseshoe holes in the temporal quadrant and was treated by too strong applications. The retina became detached, and in spite of repeated surgery a massive vitreous retraction developed. The flat detached retina in the first case and the strong extensive application in the second case were presumably the causes of the failure.

The 2 other cases developed new breaks far away from the previously treated holes. The new breaks appeared 2 and 3 years respectively after the first treatment. These were fellow-eyes with myopia of -10.0 and -20.0 dioptres. In these 2 cases we assume that vitreoretinal adhesion and traction present from the previous treatment enhanced the development of retinal detachment. Both of them became reattached after surgery.

Cases of localised retinal detachment, multiple retinal breaks with extensive applications, or vitreoretinal adhesion after repeated treatments are in our opinion unsuitable for photocoagulation, and preventive surgery has to be considered.

Since our results matched well with comparable series reported by others (Robertson and Norton, 1973, Kanski and Daniel, 1975), we believe that photocoagulation is a method as good as other methods used today. A more careful selection of cases, together with more precautions during treatment, might possibly have further reduced the number of complications.

\footnotetext{
References

Byer, N. E. (1967). Clinical study of retinal breaks. Transactions of the American Academy of Ophthalmology and Otolaryngology, 71, 461.
} 
Chignell, A. H., and Shilling, J. (1973). Prophylaxis of retinal detachment. British Journal of Ophthalmology, 57, 291.

Kanski, J. J., and Daniel, R. (1975). Retinal detachment prophylaxis. American Journal of Ophthalmology, 79, 197.

Lincoff, H. A. (1961). Archives of Ophthalmology, 66, 48.

Merin, S., Feiler, V., Hyams, S., Ivri, M., Krakowsky, D., Landau, L.: Meythar, B., Michaelson, I. C., Scharf, J., Schul, A., and Ser, J. (1971). The fate of the fellow-eye in retinal detachment. American Journal of Ophthalmology, 71, 477-481.
Neumann, E., Hyams, S., Barkai, S., Feiler, V., Ivri, M., Krakovsky, D., Meythar, B., Scharf, J., and Jedvab, E. (1972). The natural history of retinal detachment and the time factor involved. In Proceedings of the Jerusalem Seminar of Prevention of Blindness, pp. 404-408. Academic Press: New York.

Robertson, D. M., and Norton, E. D. W. (1973). Longterm follow-up of treated retinal breaks. American Journal of Ophthalmology, 75, 395. 